\title{
Oral health, dental prophylaxis and catheter-related bloodstream infections in home parenteral nutrition patients: results of a UK survey and cohort study
}

\author{
A. M. Lee ${ }^{1}$, S. M. Gabe ${ }^{2}$ J. M. Nightingale ${ }^{3}$, J. Fiske ${ }^{4}$ and M. Burke ${ }^{5}$ \\ ${ }^{1}$ North West London Hospitals NHS Trust HAI 3UJ, UK, ${ }^{2}$ Consultant Gastroenterologist \& Co-Chair of the Lennard-Jones \\ Intestinal Failure Unit, St Mark's Hospital HA1 3UJ, Honorary Senior Lecturer, Imperial College, ${ }^{3}$ Consultant \\ Gastroenterologist \& Co-Chair of the Lennard-Jones Intestinal Failure Unit, St Mark's Hospital HA1 3UJ, UK, ${ }^{4}$ Senior \\ Lecturer/Honorary Consultant in Special Care Dentistry and ${ }^{5}$ Consultant in Special Care Dentistry, Guys and St Thomas' \\ NHS Foundation Trust, London SE1 9R, UK
}

There are concerns that catheter-related bloodstream infections (CRBSI) arise from poor oral health and there are no standard guidelines for antibiotic prophylaxis for dental treatment in home parenteral nutrition (HPN) patients.

1. An email survey of the UK HPN group requesting provider's opinions, observations and prescribing practices related to oral health and CRBSI.

2. Review of data from 52 HPN patients comparing oral health parameters and dental treatment in relation to CRBSI experience in the last 12 months; using Chi-square analysis to assess associations.

3. Sixty-eight percent responded. Eighty-nine percent linked dental health/treatment with a CRBSI and $61 \%$ recommended parenteral prophylactic antibiotics (82\% IV, $18 \%$ IM). Streptococci, bacteroides and fuscobacteria caused most concern. Amoxicillin, metronidazole, co-amoxyclav and gentamycin are most prescribed. Many practitioners followed the historic infective endocarditis (IE) dental treatment guidelines, although regimes varied from current IE guidance. Thirty-six percent may delay HPN if oral health was poor; $89 \%$ believe a new central venous catheter should not delay dental treatment; $89 \%$ could not recall dental treatment impacting on HPN; and $79 \%$ had no recall of oral health impacting on HPN. Fifty-seven percent recommended dental examination or oral health screening, and $25 \%$ dental extractions, to prevent or treat CRBSI. Seventy-one percent believed patient care should be in a specialist setting.

4. There was no significant link between dental plaque, the interval since the last dental treatment or antibiotic prophylaxis and CRBSI over the previous 12 months.

Despite no evidence to support an association between dental health/treatment and CRBSI, UK HPN providers tend to prescribe prophylactic antibiotics for dental treatment; a multi-centre study would provide more robust evidence to help establish valid guidelines. 